\title{
Carcinoma basocelular de piel en el área metropolitana de Bucaramanga, Colombia: una mirada epidemiológica
}

Basal cell carcinoma in the metropolitan area of Bucaramanga, Colombia. An epidemiological view.

\section{Claudia Janeth Uribe', Katty Camila Anaya², Adriana Marcela Céspedes², Cinthya Rosa Otero², Daniela María Prada², Jennifer Paola Rueda², Tatiana Sánchez ${ }^{3}$}

1. Médica patóloga, dermatopatóloga; directora, Registro Poblacional de Cáncer del Área Metropolitana de Bucaramanga; profesora asociada, Facultad de Ciencias de la Salud, Universidad Autónoma de Bucaramanga, Colombia

2. Facultad de Ciencias de la Salud, Medicina, Universidad Autónoma de Bucaramanga, Colombia

3. Médica dermatóloga, Universidad Autónoma de Bucaramanga, Colombia.

\section{RESUMEN}

Introducción. El carcinoma basocelular es el tumor maligno más frecuente de la piel. En los últimos años se ha visto un aumento en su incidencia y, además, un mayor número de casos atípicos.

Objetivo. Describir el comportamiento epidemiológico del carcinoma basocelular en el Área Metropolitana de Bucaramanga en el año 2014.

Métodos. Se llevó a cabo un estudio poblacional observacional, retrospectivo y de corte transversal, de casos de carcinoma basocelular diagnosticados por histopatología, desde el $1^{\circ}$ de enero hasta el 31 de diciembre del 2014, en personas residentes en el Área Metropolitana de Bucaramanga.

Resultados. Se registraron 1.669 pacientes con carcinoma basocelular diagnosticados por histopatología, con una media de edad de 68,2 años. El 54,5 \% fueron mujeres, y el grupo etario más afectado el de 70 a 79 años $(26,6 \%)$.

La tasa de incidencia estandarizada fue de 124,2 por 100.000 personas por año para la población general, discriminada en 118,2 mujeres y 133 hombres por 100.00o personas por año.

La cara y el cuello fueron los sitios más comprometidos por el tumor (86,8\%), y los tipos histopatológicos más frecuentes fueron el sólido y el mixto.

Conclusiones. La incidencia del carcinoma basocelular en el área estudiada mostró una tendencia a aumentar entre el 2000 y el 2014, sin cambios en su distribución. Llama la atención el hallazgo de casos atípicos de carcinoma basocelular en zonas de la piel no expuestas al sol y sitios especiales, así como el predominio de casos en hombres menores de 40 años.

PALABRAS CLAVE: carcinoma basocelular; epidemiología; neoplasias cutáneas.
Correspondencia:

Katty Camila Anaya-Reyes

Email:

kanaya@unab.edu.co

Recibido: 22/04/17

Aceptado: 22/11/17

Conflictos de interés:

No se reportan conflictos de interés.

\section{Financiación:}

Ninguna. 


\section{SUMMARY}

Introduction. The basal cell carcinoma is the most frequent malignant tumor of the skin, in the last years there has been an increase in its incidence and a greater number of atypical cases.

Objective: To describe the epidemiological behavior of the basal cell carcinoma in the Metropolitan Area of Bucaramanga for the year 2014 .

Methods: A retrospective population-based study of cases of basal cell carcinoma diagnosed by histopathology from January 1st to December 31st of 2014, in patients residents of the Metropolitan Area of Bucaramanga.

Results: One-thousand six-hundred and sixty-nine patients were recruited with basal cell carcinoma diagnosed by histopathology, with an average age of 68.2 years. Fifty-four point six per cent of the patients were women, and the most frequent age group was 70-79 years (26.6\%). The standardized incidence rate was 124.2 per 100,000 people per year for the general population, with a women' standardized incidence rate of 118.2 per 100,000 people per year, and for men of 133 per 100.000 people per year. The face and neck were the most affected areas (86.8\%), and the most common histopathological types were solid and mixed.

Conclusions: The incidence of basal cell carcinoma in the Metropolitan Area of Bucaramanga has shown an increase in the incidence from 2000 to 2014 year, without changes in its distribution. It draws attention to the finding of atypical cases of basal cell carcinoma in areas not exposed to light and special locations, as well as the predominance of cases in men under 40 years of age.

KEY WORDS: Basal cell carcinoma; epidemiology; skin neoplasms.

\section{" Por sus características geográficas y por el predominio de fototipos de piel de bajo grado en su población, Australia es el país con mayor incidencia de carcinoma basocelular en el mundo, con una tasa de incidencia estandarizada de 884 por 100.000 personas por año."}

\section{INTRODUCCIÓN}

El carcinoma de piel es la neoplasia maligna más común de todas y se clasifica como cáncer de piel de tipo melanoma y no melanoma. Se calcula que hay entre 2 y 3 millones de casos de cáncer de piel no melanoma en el mundo ${ }^{(1)}$, y el $80 \%$ de ellos corresponden a carcinoma basocelular ${ }^{(2)}$, el cual está relacionado principalmente con la exposición a los rayos ultravioleta.

Por sus características geográficas y por el predominio de fototipos de piel de bajo grado en su población, Australia es el país con mayor incidencia de carcinoma basocelular en el mundo, con una tasa de incidencia estandarizada de 884 por 100.000 personas por año ${ }^{\text {(3) }}$. Por su ubicación ecuatorial, Colombia está expuesta a altos niveles de radiación ultravioleta (UV), principalmente UVB. La radiación UVA y la UVB se relacionan con lesiones por estrés por fotooxidación y mutaciones, la segunda con un mayor daño directo en el ADN celular, efectos que favorecen el desarrollo de cáncer de piel (4-6). En Colombia, no se encuentran otros reportes de datos de incidencia de carcinoma basocelular distintos a los del Registro Poblacional de Cáncer del Área Metropolitana de Bucaramanga, que permitan conocer su tendencia en el tiempo, por lo que este estudio busca describir el comportamiento epidemiológico de esta neoplasia en el Área Metropolitana de Bucaramanga durante el año 2014 .

\section{MATERIALES Y MÉTODOS}

Se llevó a cabo un estudio poblacional, observacional, retrospectivo y de corte transversal, con inclusión de 1.669 casos con diagnóstico histopatológico de carcinoma basocelular del $1^{\circ}$ de enero al 31 de diciembre del 2014, en pacientes residentes del Área Metropolitana de Bucaramanga.

El Registro Poblacional de Cáncer del Área Metropoli- 
tana de Bucaramanga tuvo acceso a la información de todos los laboratorios de patología de esta localidad. Este registro poblacional de cáncer es uno de los cinco en Colombia avalados por la International Association of Cancer Registries de la International Agency for Research in Cancer (IARC) de la Organización Mundial de la Salud (OMS), y que hacen parte del Sistema Nacional de Información en Cáncer, el Observatorio Nacional de Cáncer y el Sistema de Vigilancia en Salud Pública por la Resolución $N^{\circ} 01281^{(7)}$, y de la Ley Sandra Ceballos 1348 del 19 de abril del $2010^{(8)}$, del Ministerio de Salud y la Protección Social de Colombia. Este registro viene funcionando de forma permanente desde el año 2000, como proyecto de investigación de la Universidad Autónoma de Bucaramanga (UNAB) y con el aval de su Comité de Ética.

Para la codificación de cada caso se utilizó la tercera edición de la Clasificación Internacional de Enfermedades Oncológicas (CIE-O) ${ }^{9}$ ). La información demográfica se validó utilizando bases de datos gubernamentales disponibles en la red, así como bases de datos de comprobador de derechos de la Secretaría de Salud Departamental. La información fue digitada y analizada con el software CANREG5, y se hicieron análisis adicionales en Excel®. Se siguieron protocolos para garantizar la confidencialidad de los datos personales.

La información de la población proyectada para el año 2014, por sexo y grupos quinquenales de edad, se obtuvo del Departamento Administrativo Nacional de Es- tadística (DANE) ${ }^{(10)}$. Para el cálculo de las tasas de incidencia estandarizada, se usó el estándar de la población mundial 'Segi' (world) ${ }^{(11)}$.

\section{RESULTADOS}

De los 1.669 pacientes con diagnóstico de carcinoma basocelular, el 54,5 \% fueron mujeres, con una relación mujer a hombre de 1,2:1. La tasa de incidencia estandarizada fue de 124,2 por 100.000 personas por año en la población general y, discriminada por sexo, de 118,2 mujeres y 133 hombres por 100.000 personas por año (tabla1).

El carcinoma basocelular se diagnosticó entre los 17 y los 99 años de edad, con una edad media de 68,2 años; el mayor número de casos ocurrió en el grupo de 70 a 79 años (26,6 \%), seguido del de 60 a 69 años (23,4\%). En casi todos los grupos etarios hubo mayor compromiso en las mujeres, excepto en el de menores de 40 años donde los hombres representaron el 2,4\% y las mujeres solo el $0.1 \%$ de los casos (tabla 1).

La cara y el cuello fueron los sitios más comprometidos $(86,8 \%)$, especialmente la nariz (40,5\%), seguida de los párpados (12,6\%) y las mejillas (12\%). El 7,9\% de los casos ocurrió en áreas de la piel no expuestas al sol y el o,3 \% correspondió a áreas especiales (axilas, ingle, pliegues submamarios, vagina y senos). La variante histopatológica más frecuente fue el tipo sólido, prin-

Tabla 1. Tasas de incidencia y grupos de edad por sexo, Registro Poblacional de Cáncer del Ârea Metropolitana de Bucaramanga, 2014

\begin{tabular}{|c|c|c|c|c|c|c|}
\hline \multirow{2}{*}{$\begin{array}{c}\text { GRUPOS } \\
\text { DE EDAD } \\
\text { (años) }\end{array}$} & \multicolumn{4}{|c|}{ SEXO } & \multicolumn{2}{|c|}{ Total } \\
\hline & n (\%) & TEE & $n(\%)^{a}$ & TEE & n (\%) & TEE \\
\hline $10-19$ & o (o) & 0 & $2(0,1)$ & 0,38 & $2(0,1)$ & 0,19 \\
\hline $20-29$ & o (o) & 0 & $3(0,1)$ & 0,50 & $3(0,1)$ & 0,25 \\
\hline $30-39$ & $2(0,1)$ & 0,28 & $37(2,2)$ & 5,41 & $39(2,3)$ & 2,78 \\
\hline $40-49$ & $86(5,1)$ & 12,98 & $49(2,9)$ & 8,58 & $135(8,0)$ & 10,95 \\
\hline $50-59$ & $156(9,4)$ & 20,79 & $118(7,1)$ & 19,02 & $274(16,4)$ & 19,99 \\
\hline $60-69$ & $217(13,0)$ & 36,50 & $174(10,4)$ & 37,12 & $391(23,4)$ & 36,78 \\
\hline $70-79$ & $236(14,1)$ & 29,30 & $209(12,5)$ & 37,46 & $445(26,6)$ & 32,64 \\
\hline$>80$ & $214(12,8)$ & 18,41 & $166(10)$ & 24,57 & $380(22,7)$ & 20,68 \\
\hline Total & $911(54,5)$ & 118,28 & $758(45,5)$ & 133,04 & $1669(100)$ & 124,26 \\
\hline
\end{tabular}


cipalmente en el cuero cabelludo, seguida de variantes más agresivas, como el tipo mixto en los miembros inferiores y el micronodular en el tronco, la cara y el cuello (tabla 2) (figura 1).

\section{DISCUSIÓN}

El conocimiento de cifras de incidencia de cáncer de una población se obtiene mediante los casos observados y consignados en los registros poblacionales de cáncer (12) o por medio de proyecciones que, para Colombia, las hace el Instituto Nacional de Cancerología.

En el país, los registros poblacionales de cáncer no recopilan de forma rutinaria la información de los casos de cáncer de piel de tipo no melanona. La única referencia relacionada con datos de carcinoma basocelular en los últimos 15 años, la tiene el Registro Poblacional de Cáncer del Área Metropolitana de Bucaramanga
(13). Algunas Instituciones Prestadoras de Servicios de Salud (IPS) en el país han publicado datos sobre esta enfermedad, poniendo en evidencia el problema desde el punto de vista de la atención en salud, pero sin que se logre conocer así la incidencia en la población nacional $^{(12)}$.

Por lo anterior, y al comparar los resultados de la misma población a los 13 años del estudio inicial (2000-2001), se observó un aumento de la tasa de incidencia estandarizada en 20,7 casos por 100.000 personas al año, lo que da un aumento aproximado de 1,5 casos por 100.000 personas por año ${ }^{(13)}$. Esta tendencia al aumento del carcinoma basocelular en el tiempo, ha sido reportada en otros países como Australia (país con mayor incidencia mundial de carcinoma basocelular) donde, como es de esperarse, ha tenido un incremento mucho mayor (13,3 casos por 100.000 personas por año por cada año del análisis (1985 al 2002) ${ }^{(14)}$. Asimismo, en otras referencias institucionales de Colombia y Latinoamérica, se ha pu-

Tabla 2. Sitio anatómico y tipo histopatológico por sexo, Registro Poblacional

de Cáncer del Ârea Metropolitana de Bucaramanga, 2014

\begin{tabular}{|c|c|c|c|c|c|c|}
\hline \multirow{3}{*}{$\begin{array}{c}\text { SITIO } \\
\text { ANATÓMICO }\end{array}$} & \multicolumn{4}{|c|}{ SEXO } & \multirow{2}{*}{\multicolumn{2}{|c|}{ Total }} \\
\hline & \multicolumn{2}{|c|}{ Mujeres } & \multicolumn{2}{|c|}{ Hombres } & & \\
\hline & $n$ & $(\%)^{\mathrm{a}}$ & $n$ & $(\%)^{\mathrm{a}}$ & $\mathbf{n}$ & $(\%)^{\mathrm{a}}$ \\
\hline Cuero cabelludo & 14 & 1.5 & 16 & 2.1 & 30 & 1.8 \\
\hline Cara y cuello & 800 & 87.8 & 648 & 85.5 & 1.448 & 86.8 \\
\hline Miembro superior proximal & 17 & 1.9 & 17 & 2.2 & 34 & 2.0 \\
\hline Miembro superior distal & 14 & 1.5 & 8 & 1.1 & 22 & 1.3 \\
\hline Tronco & 31 & 3.4 & 50 & 6.6 & 81 & 4.9 \\
\hline Miembros inferiores & 9 & 1 & 3 & 0.4 & 12 & 0.7 \\
\hline Áeas especiales ${ }^{b}$ & 4 & 0.5 & 1 & 0.1 & 5 & 0.3 \\
\hline Desconocido & 22 & 2.4 & 15 & 2 & 37 & 2.2 \\
\hline \multicolumn{7}{|l|}{ Tipo hispatológico } \\
\hline Sólido & 543 & 59.6 & 464 & 61.2 & 1.007 & 60.3 \\
\hline Mixto & 159 & $17 \cdot 5$ & 132 & 17.4 & 291 & $17 \cdot 4$ \\
\hline Micronodular & 76 & 8.3 & 59 & 7.8 & 135 & 8.1 \\
\hline Trabecular & 52 & 5.7 & 42 & 5.5 & 94 & 5.6 \\
\hline Superficial & 43 & 4.7 & 26 & 3.4 & 69 & 4.1 \\
\hline Desconocido & 15 & 1.7 & 13 & 1.7 & 28 & 1.7 \\
\hline Otros & 10 & 1.1 & 12 & 1.6 & 22 & 1.3 \\
\hline Morfeiforme & 11 & 1.2 & 9 & 1.2 & 20 & 1.2 \\
\hline Metatípico & 2 & 0.2 & 2 & 0.2 & 4 & 0.2 \\
\hline Total & 911 & 100 & 758 & 100 & 1669 & 100 \\
\hline
\end{tabular}

a Porcentaje del total de los casos

${ }^{\mathrm{b}}$ Axilas, ingle, pliegue submamario, vagina y senos 


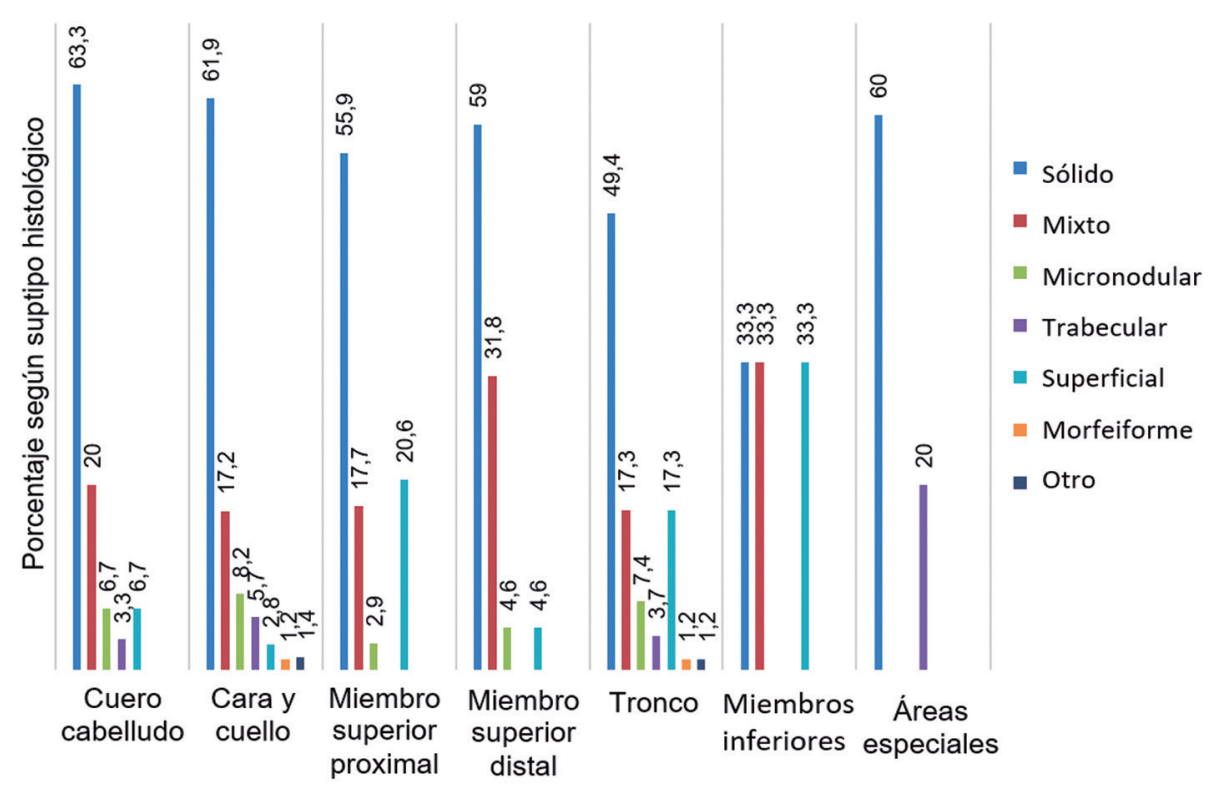

Figura 1. Distribución por localización y subtipo histológico

blicado este incremento en la incidencia de carcinoma basocelular ${ }^{(6,15-18)}$.

El aumento en la incidencia de esta neoplasia en el Área Metropolitana de Bucaramanga desde el 2000 hasta el 2014, no ha mostrado variación al discriminarla según el sexo, y continúa siendo más frecuente en las mujeres, en contraste con Australia y Norteamérica, donde los hombres aportan el mayor número de $\operatorname{casos}^{(14,19,20)}$.

En el área estudiada, la incidencia discriminada según el sitio anatómico comprometido no ha variado en el tiempo, como tampoco lo ha hecho en otros países como Estados Unidos y Australia ${ }^{(14,20)}$. No obstante, sí ha aumentado en los sitios anatómicos no expuestos regularmente al sol: en los hombres, el tronco, y en las mujeres, los miembros inferiores; hallazgo que es similar en las poblaciones de Alemania y Australia ${ }^{(14,21)}$.

Llama la atención el comportamiento de la enfermedad en los menores de 40 años, de los cuales el 95,5\% de los casos se presentó en hombres. Lo contrario sucedió en los años 2000 y 2001, cuando no hubo una diferencia significativa según el sexo (54,3 \% en mujeres y 45,6 \% en hombres), similar a lo informado en Australia donde este porcentaje en hombres fue de $69,5 \%{ }^{(19,20)}$.

\section{CONCLUSIONES}

La incidencia del carcinoma basocelular en el Área Metropolitana de Bucaramanga ha mostrado una ten- dencia al aumento entre el 2000 y el 2014, sin cambios en su distribución según edad, sexo, localización o tipo histológico. Llama la atención el hallazgo de casos atípicos de carcinoma basocelular en áreas no expuestas al sol y en sitios especiales, así como el predominio de casos en hombres menores de 40 años.

\section{REFERENCIAS}

1. World Health Organization. Ultraviolet radiation. Skin cancers. Fecha de consulta: Febrero 14 de 2017. Disponible en: http://www.who.int/uv/faq/ skincancer/en/index1.html.

2. American Cancer Society. Key statistics for basal and squamous cell skin cancers; 2016. Fecha de consulta: Febrero 14 de 2017. Disponible en: https://www.cancer.org/cancer/basal-and- squamous-cell- skin-cancer/about/key-statistics.html.

3. Australian Institute of Health and Welfare. Skin cancer in Australia; 2016. Fecha de consulta: Febrero 14 de 2017. Disponible en: https://www. aihw.gov.au/reports/cancer/skin-cancer-in-australia/contents/table-of-contents

4. Pfeifer GP, Besaratinia A. UV wavelength-dependent DNA damage and human nonmelanoma and melanoma skin cancer. Photochem Photobiol. 2012;11:90-7.

5. Sanchez G, Nova J, Hoz F de la. Risk Factors for 
Squamous Cell Carcinoma, a study by the National Dermatology Centre of Colombia. Actas Dermosifiliogr. 2012;103:294-300.

6. Sanchez G, Nova J, Hoz F de la, Castañeda C. Incidencia de cáncer de piel en Colombia, años 20032007. Piel. 2011;26:171-7.

7. Ministerio de Salud y Protección Social. Serie: Registros, observatorios, sistemas de seguimiento y salas situacionales nacionales en Salud ROSS. En: Observatorio Nacional de Cáncer, guía metodologica; 2015. p.28-30. Fecha de consulta: Marzo o3 de 2017. Disponible en: https://www.minsalud. gov.co/sites/rid/Lists/BibliotecaDigital/RIDE/VS/ ED/GCFI/guia-ross- cancer.pdf.

8. Ley Sandra Ceballos. "Por lo cual se establecen las acciones para la atención integral del cáncer en Colombia". Ley No.1384 del 19 de abril del 2010. Articulo $\mathrm{N}^{\circ}{ }_{15}$. Fecha de consulta: Marzo 03 de 2017. Disponible en: http://www.ins.gov.co/normatividad/Leyes/LEY 1384 DE 2010.pdf.

9. Fritz A, Percy C, Jack A, Shanmugaratnam K, Sobin L, Parkin DM, et al. Clasificación Internacional de Enfermedades para Oncología. Tercera edición. Washington D.C. Organización Panamericana de la Salud. 2003. p.32

10. Departamento Administrativo Nacional de Estadística (DANE). Demografía y Población - Proyecciones de Población. Fecha de consulta: Marzo 22 de 2017. Disponible en: https://www.dane.gov.co/ index.php/estadisticas-por-tema/demografia-ypoblacion/proyecciones-de- poblacion.

11. Ahmad OB, Boschi-Pinto C, Lopez AD, Murray CJ, Lozano R, Inoue M. Age standardization of rates: A new WHO standard. World Health Organization (GPE Discuss Pap Ser). 2001;31:1-14. Fecha de consulta: Marzo 22 de 2017. Disponible en: http:// www.who.int/healthinfo/paper31.pdf.

12. Bray F, Znaor A, Cueva P, Korir A, Swaminathan R, Ullrich A, et al. Planificación y desarrollo de registros de cáncer de base poblacional en los países de ingresos bajos y medios. Publicaciones técnicas $\mathrm{N}^{\circ}$ 43. Lyon: IARC; 2015.

13. Uribe C, Meza E, Ávila M. Epidemiología del carcinoma basocelular. Análisis de 1.423 casos observados en la población del área metropolitana de Bucaramanga. Basado en el registro poblacional de cáncer. Rev Asoc Col Dermatol. 2007;15:275-9.

14. Staples MP, Elwood M, Burton RC, Williams JL, Marks R, Giles GG. Non-melanoma skin cancer in Australia: The 2002 national survey and trends since 1985. Med J Aust. 2006;184:6-10.

15. Nova-Villanueva J, Sánchez-Vanegas G, Porras De
Quintana L. Cáncer de Piel: Perfil epidemiológico de un centro de referencia en Colombia 2003-2005. Rev Salud Pública. 2007;9:595-601.

16. Nova J, Patiño A, González A. Caracterización de la población con carcinoma basocelular en el Centro Dermatológico Federico Lleras Acosta. Rev CES Med. 2014;28:177-84.

17. Cormane J, Rodelo A. Epidemiología del cáncer no melanoma en Colombia. Rev Asoc Col Dermatol. 2014;22:20-6.

18. Hernández-Zárate SI, Medina-Bojórquez A, LópezTello Santillán L, Alcalá-Pérez D. Epidemiología del cáncer de piel en pacientes de la clínica de dermato-oncología del Centro Dermatológico Dr. Ladislao de la Pascua. Estudio retrospectivo de los últimos ocho años. Dermatologia Rev Mex. 2012;56:30-7.

19. Richmond-Sinclair NM, Pandeya N, Ware RS, Neale RE, Williams GM, van der Pols JC, et al. Incidence of basal cell carcinoma multiplicity and detailed anatomic distribution: Longitudinal study of an australian population. J Invest Dermatol. 2009;129:323-8.

20. Eide MJ, Weinstock MA. Epidemiology of skin cancer. En: Rigel DS, Robinson JK, Ross M, Friedman RJ, Cockerell CJ, Lim HW, et al., editors. Cancer of the skin. Second edition. Elsevier Sounders; 2011. p.44-55.

21. Katalinic A, Kunze U, Schäfer T. Epidemiology of cutaneous melanoma and non-melanoma skin cancer in Schleswig-Holstein, Germany: Incidence, clinical subtypes, tumour stages and localization (epidemiology of skin cancer). Br J Dermatol. 2003;149:1200-6. 